Rapid Communications

\title{
Regioselective Immobilization of a PVC Membrane Composed of an Ionic Liquid-based Dye on Convex-shaped PDMS Surface for Multiplexed Microanalytical Devices
}

\author{
Tatsumi Mizuta, Kenichi Maeno, Kenji Sueyoshi, Tatsuro Endo, and Hideaki Hisamoto ${ }^{\dagger}$ \\ Department of Applied Chemistry, Graduate School of Engineering, Osaka Prefecture University, 1-1 Gakuen-cho, \\ Naka, Sakai, Osaka 599-8531 Japan
}

\begin{abstract}
A novel method for the intact immobilization of a very-thin and soft PVC membrane on a convex-shaped poly(dimethylsiloxane) (PDMS) surface is described. The present method using PVA film as a sacrificial layer allowed successful immobilization of an intact PVC membrane using an ionic liquid-based dye on only the convex-shaped PDMS surface without any deformation or increase of the inhomogeneity. In addition, two different kinds of PVC membranes were successfully immobilized simultaneously toward multiplexed detection.
\end{abstract}

Keywords Ionic liquid, optical sensor, plasticized poly(vinyl chloride) membrane, microanalytical devices

(Received April 2, 2018; Accepted April 11, 2018; Published May 10, 2018)

Poly(dimethylsiloxane) (PDMS) is one of the most common microfluidic substrates due to its reasonable cost, rapid prototyping capability, and ease of implementation. ${ }^{1}$ Today, many applications of microfluidic devices based on PDMS have been developed for chemical analysis and bioassays. ${ }^{2-11}$

We have been developing a combinable PDMS capillary (CPC) sensor array fabricated by combining convex- and concave-shaped PDMS plates. ${ }^{12-14}$ In this case, a single-step analysis of various analytes was successfully demonstrated by independently immobilizing various analytical reagents or coatings on the convex- or concave-shaped PDMS surfaces. On the other hand, we have been focusing on the use of plasticized poly(vinyl chloride) (PVC) membranes for capillary-based microanalytical devices, due to their versatility in analytical applications, such as multiplexed ion sensing, ${ }^{15,16}$ enzyme biosensing, ${ }^{17}$ or single-step immunoassay. ${ }^{18}$

Very recently, we have developed a plasticizer-free PVC membrane composed of PVC and ionic liquid-based dye, which was called a "dyed plasticizer" due to its dual functions of color-change response and plasticization. ${ }^{19}$ This PVC membrane could contain an extremely high concentration of dye because a conventional plasticizer was not needed. In this study, very fast and highly sensitive anion sensing was successfully achieved due to the use of a very thinner $(<1 \mu \mathrm{m})$ membrane and a higher concentration of dye compared with conventional PVC membranes. Therefore, combining PVC membrane using an ionic liquid-based dye and a CPC sensor array would be expected to open a new field of applications in the telemedicine field where the rapid and simultaneous detection of various disease markers by the naked eye at home is strongly desired. However, since a PVC membrane using an ionic liquid-based dye is too thin (in some cases, less than $200 \mathrm{~nm}$ ) to handle, it is very difficult to control the immobilizing position on a substrate

† To whom correspondence should be addressed.

E-mail: hisamoto@chem.osakafu-u.ac.jp without any deformation or increase in the inhomogeneity of the PVC membrane.

Herein, we developed a novel immobilization method using poly(vinyl alcohol) (PVA) film as a sacrificial layer ${ }^{20}$ and a PET film mask. In this case, the lamination of a PVC membrane spin-coated on a PVA film, a PET film mask and a convexshaped PDMS plate, followed by dissolution of the PVA layer by a water rinse, and subsequent removal of the PET film mask allowed the successful immobilization of a very thin and soft PVC membrane (Fig. 1). In addition, we successfully achieved the immobilization of different membranes on the same PDMS substrate by the present method.

The fabrication procedure of a convex-shaped PDMS is shown in Supporting Information. Here, we used a pillar array structure of $3 \mathrm{~mm}$ diameter and $120 \mu \mathrm{m}$ height, and tried to immobilize only on the top surface of the pillars. As the polymer liquid membrane, a PVC membrane composed of PVC and an ionic liquid-based dye, $\left[\mathrm{P}_{66614}\right]_{2}[\mathrm{NP}],{ }^{19}$ was used. The PVC membrane was prepared with a THF solution of PVC and $\left[\mathrm{P}_{66614}\right]_{2}[\mathrm{NP}](1: 9$ (wt/wt)) on a PVA film (15 $\mu \mathrm{m}$ thickness) by spin-coating (1600 rpm, $20 \mathrm{~s})$. The appearance of the PVC membrane spincoated on the PVA film is shown in Fig. S5(i) in Supporting Information. A PET film mask (100 $\mu \mathrm{m}$ thickness) was adhered to the PDMS substrate by manual handling. In this case, surface treatments of the PDMS substrate and PET film were not carried out. The PVC side of the PVC membrane on PVA film was attached to a convex-shaped PDMS substrate masked with PET film, and those were immersed in distilled water $(30 \mathrm{~min})$. Afterward, the PET film mask was removed, and then a convexshaped PDMS substrate immobilizing the PVC membrane on the top surface of pillar structures was prepared (Fig. 2, Fig. S4, Supporting Information). In this case, the PVC membrane adhered onto the PDMS surface was not removed together with the PET film mask because of strong adhesion of the PVC membrane on the PDMS surface due to its own high hydrophobicity. By using this method, since a PVC membrane 

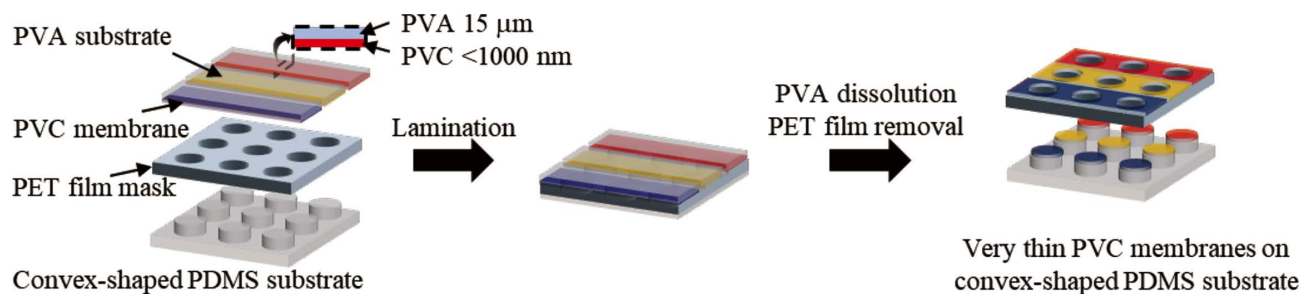

Fig. 1 Proposed method for immobilizing a PVC membrane on PDMS substrate only at the top surface of a convex-shaped structurer.

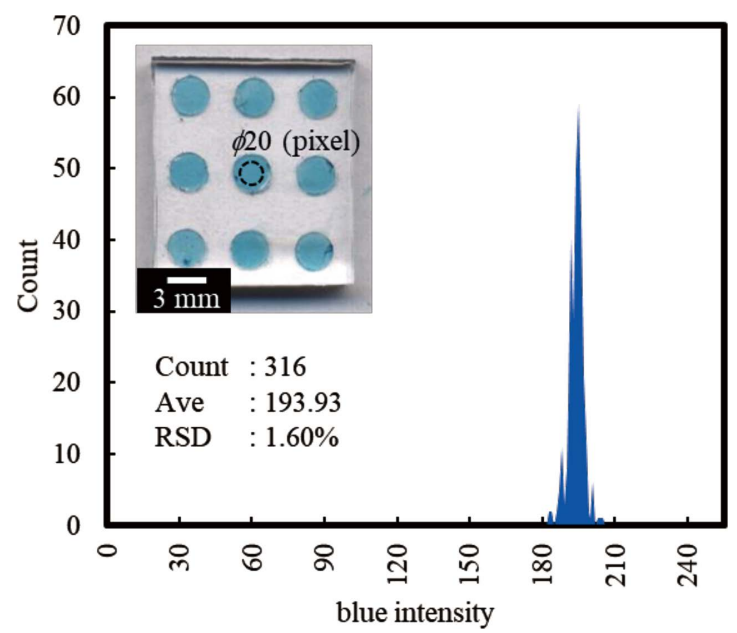

Fig. 2 Scanned image of a PDMS substrate immobilizing a PVC membrane using $\left[\mathrm{P}_{66614}\right]_{2}[\mathrm{NP}]$ (thickness, $1.0 \mu \mathrm{m}$ ) obtained by a flatbed scanner, and a normal distribution of the blue intensity of an immobilized PVC membrane on the PDMS substrate (Count, 316; Ave, 193.93; RSD, 1.60\%).

spin-coated on a PVA film can be easily handled or cut, while controlling the immobilization position is also possible. In order to investigate whether a PVC membrane could be transferred to a PDMS substrate from a PVA film homogeneously, the color distribution in the immobilized PVC membrane was evaluated. Figure 2 shows a digital image of a PVC membrane on the PDMS substrate obtained with a flat-bed scanner and a color distribution of blue intensity. In this case, the PDMS substrate side was faced downward to the scanner so that the PVC membrane side would remain intact. The relative standard deviation of the blue intensity obtained had a very small value $(1.60 \%)$, which was slightly larger value than that of a PVC membrane on a PVA substrate under the same spin-coating condition (1.29\%) (Fig. S5, Supporting Information). The obtained error was supposed to include errors by manual handling when the PVC membrane was transferred to the PDMS substrate or those by spin-coating. Although step-by-step procedures were required when the PVC membrane was transferred, the prepared PVC membrane was accurately immobilized without any deformation or increase of the inhomogeneity, because the relative standard deviation was not increased substantially. In addition, PVA dissolution-based immobilization of liquid membranes in this work has a strong advantage from the view point of membrane thickness control, which can be easily determined by the spin-coating condition, and can be transferred directly. Previously, the immobilization of a very thin and soft membrane having a constant thickness at

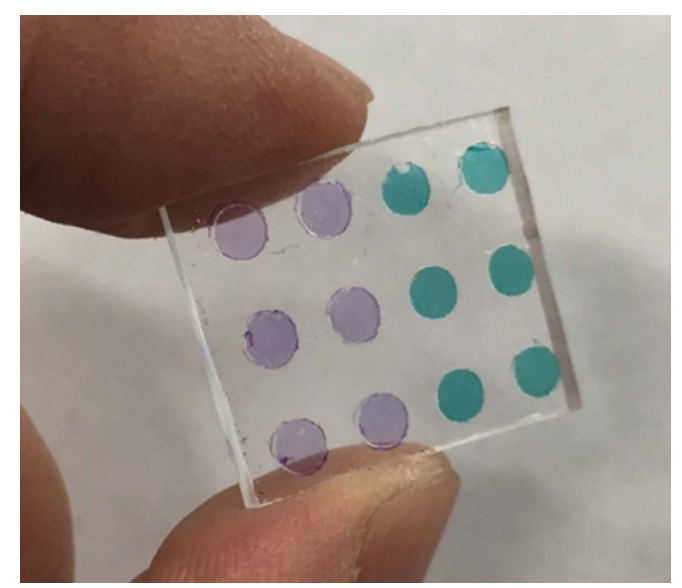

Fig. 3 Photograph of a PDMS substrate immobilizing two kinds of PVC membranes, utilizing $\left[\mathrm{P}_{66614}\right]_{2}[\mathrm{BTB}]$ (right side), and $\left[\mathrm{P}_{66614}\right]_{2}[\mathrm{PR}]$ (left side).

a specific position has been difficult. Thus, the present immobilization method can be considered to be an effective process to solve the above-mentioned difficulties. On the other hand, we also tried to immobilize the same PVC membrane by stamping a convex-shaped PDMS substrate onto a PVC membrane spin-coated on the glass substrate, like in the microcontact printing method. In this method, the immobilization of a PVC membrane was partially possible. However, due to the deformation of soft PDMS and PVC membranes during the stamping procedure, the reproducibility of the immobilization in terms of the membrane thickness or homogeneous color distribution was very bad. In addition, because the glass substrate is hard to be cut freely, regioselective immobilization is also difficult.

With the present immobilization method, the immobilization of different kinds of membranes on the same substrate can be easily carried out. In order to immobilize two different kinds of PVC membranes, PVC membranes prepared by two different kinds of "dyed plasticizer", $\left[\mathrm{P}_{66614}\right]_{2}[\mathrm{BTB}]$ and $\left[\mathrm{P}_{66614}\right]_{2}[\mathrm{PR}]$ (for synthesis and membrane composition, see text and Table S1 in Supporting Information), were prepared independently, and attached at a different position on the same (PET-masked) convex-shaped PDMS surface. Another procedure was the same as described above. Figure 3 shows a PDMS substrate immobilizing PVC membrane using $\left[\mathrm{P}_{66614}\right]_{2}[\mathrm{BTB}]$ (right side) and $\left[\mathrm{P}_{66614}\right]_{2}[\mathrm{PR}]$ (left side) respectively. This PDMS substrate was fabricated only by the simultaneous lamination of two different PVC membranes. Thus, various polymer liquid membranes can be freely immobilized on any convex-shaped parts of interest, and it is suggested that multiplexed analysis 
using various polymer liquid membranes on the same microfluidic devices can be possible.

In conclusion, we successfully developed a method that can immobilize a soft and thin liquid membrane onto a convexshaped PDMS surface. Based on this method, a polymer liquid membrane can be transferred without any deformation or increase in the inhomogeneity of membranes. In addition, different kinds of polymer liquid membranes can be easily immobilized on the same substrate simultaneously. Thus, by combining with a concave-shaped PDMS plate, immobilizing various analytical reagents ${ }^{12-14,22}$ to fabricate a novel CPC sensor array would allow us to achieve the rapid and highly-sensitive naked eye-based detection of various analytes using previously established sensing principles for plasticized PVC membranes. ${ }^{15-18,23-29}$

\section{Acknowledgements}

This work was partially supported by The Asahi Glass Foundation, Tokyo Kasei Chemical Promotion Foundation, Izumi Science and Technology Foundation, Nakatani Foundation, and Takahashi Industrial and Economic Research Foundation.

\section{Supporting Information}

This material is available free of charge on the Web at http:// www.jsac.or.jp/analsci/.

\section{References}

1. P. N. Nge, C. I. Rogers, and A. T. Woolley, Chem. Rev., 2013, 113, 2550.

2. J. de Jong, R. G. H. Lammertink, and M. Wessling, Lab Chip, 2006, 6, 1125.

3. E. Eteshola and D. Leckband, Sens. Actuators, B, 2001, 72, 129.

4. I. K. Dimov, L. Basabe-Desmonts, J. L. Garcia-Cordero, B. M. Ross, A. J. Riccoa, and L. P. Lee, Lab Chip, 2011, 11, 845.

5. P. Inpota, K. Strzelak, R. Koncki, W. Sripumkhai, W. Jeamsaksiri, N. Ratanawimarnwong, P. Wilairat, N. Choengchan, R. Chantiwas, and D. Nacapricha, Anal. Sci., 2018, 34, 161 .

6. M. A. Ashagre and T. Masadome, Anal. Sci., 2018, 34, 195.

7. R. Ishihara, Y. Uchino, K. Hosokawa, M. Maeda, and A.
Kikuchi, Anal. Sci., 2017, 33, 197.

8. A. Shirai, K. Nakashima, K. Sueyoshi, T. Endo, and H. Hisamoto, Anal. Sci., 2017, 33, 969.

9. K. Hasegawa, R. Negishi, M. Matsumoto, M. Yohda, K. Hosokawa, and M. Maeda, Anal. Sci., 2017, 33, 171.

10. M. Maeki, H. Yamaguchi, M. Tokeshi, and M. Miyazaki, Anal. Sci., 2016, 32, 3.

11. Y. Kazama and A. Hibara, Anal. Sci., 2016, 32, 99.

12. Y. Uchiyama, F. Okubo, K. Akai, Y. Fujii, T. G. Henares, K. Kawamura, T. Yao, T. Endo, and H. Hisamoto, Lab Chip, 2012, 12, 204.

13. Y. Fujii, T. G. Henares, K. Kawamura, T. Endo, and H. Hisamoto, Lab Chip, 2012, 12, 1522.

14. T. Ishimoto, K. Jigawa, T. G. Henares, T. Endo, and H. Hisamoto, Analyst, 2013, 138, 3158.

15. H. Hisamoto, Y. Nakashima, C. Kitamura, S. Funano, M. Yasuoka, K. Morishima, Y. Kikutani, T. Kitamori, and S. Terabe, Anal. Chem., 2004, 76, 3222.

16. H. Hisamoto, M. Yasuoka, and S. Terabe, Anal. Chim. Acta, 2006, 556, 164.

17. E. Tsutsumi, T. G. Henares, K. Kawamura, T. Yao, and H. Hisamoto, Chem. Lett., 2010, 39, 436.

18. S. Funano, M. Sugahara, T. G. Henares, K. Sueyoshi, T. Endo, and H. Hisamoto, Analyst, 2015, 140, 1459.

19. T. Mizuta, K. Sueyoshi, T. Endo, and H. Hisamoto, Sens. Actuators, B, 2018, 258, 1125.

20. S. Aki, T. Endo, K. Sueyoshi, and H. Hisamoto, Anal. Chem., 2014, 86, 11986.

21. W. I. S. Galpothdeniya, K. S. McCarter, S. L. De Rooy, B. P. Regmi, S. Das, F. Hasan, A. Tagge, and I. M. Warner, RSC Adv., 2014, 4, 7225.

22. H. Wakayama, T. G. Henares, K. Jigawa, S. Funano, K. Sueyoshi, T. Endo, and H. Hisamoto, Lab Chip, 2013, 13, 4304.

23. E. Bakker, P. Buhlmann, and E. Pretsch, Chem. Rev., 1997, 97, 3083.

24. P. Buhlmann, E. Pretsch, and E. Bakker, Chem. Rev., 1998, 98, 1593.

25. J. Zhai, X. Xie, and E. Bakker, Anal. Chem., 2015, 87, 2827.

26. A. S. Watts, A. A. Urbas, E. Moschou, V. G. Gavalas, J. V. Zoval, M. Madou, and L. G. Bachas, Anal. Chem., 2007, $79,8046$.

27. Z. Jarolímová, M. Vishe, J. Lacour, and E. Bakker, Chem. Sci., 2016, 7, 525.

28. R. D. Johnson, I. H. A. Badr, G. Barrett, S. Lai, Y. Lu, M. J. Madou, and L. G. Bachas, Anal. Chem., 2001, 73, 3940.

29. I. H. A. Badr, R. D. Johnson, M. J. Madou, and L. G. Bachas, Anal. Chem., 2002, 74, 5569. 